Résumés des conférences et travaux

\title{
Langue et littérature néo-latines
}

\section{Perrine Galand}

\section{(2) OpenEdition Journals}

Édition électronique

URL : https://journals.openedition.org/ashp/1157

DOI : 10.4000/ashp.1157

ISSN : 1969-6310

Éditeur

Publications de l'École Pratique des Hautes Études

\section{Édition imprimée}

Date de publication : 1 octobre 2011

Pagination : 119-121

ISSN : 0766-0677

\section{Référence électronique}

Perrine Galand, « Langue et littérature néo-latines », Annuaire de l'École pratique des hautes études (EPHE), Section des sciences historiques et philologiques [En ligne], 142 | 2011, mis en ligne le 25 juillet 2011, consulté le 06 juillet 2021. URL : http://journals.openedition.org/ashp/1157 ; DOI : https:// doi.org/10.4000/ashp. 1157 


\title{
LANGUE ET LITTÉRATURE NÉO-LATINES
}

\author{
Directeur d'études : M ${ }^{\text {me }}$ Perrine Galand
}

Programme de l'année 2009-2010 : I. Théories et pratiques poétiques de l'humanisme (XVI siècle) : la poésie (latine et française) des Parlementaires, dans la seconde moitié du $X V I^{e}$ siècle en France (le statut de leur activité poétique; une tradition érasmienne tardive; la question du bilinguisme), suite : les Poemata d'Étienne de La Boétie (Paris, Federic Morel, 1571). - II. Édition, traduction et commentaire de textes humanistes: John Nassichuk (professeur à l'université de Western Ontario, Canada) : Les Poemata (Caen, 1600) de l'humaniste Jean Rouxel de Breteuil, professeur de droit et de rhétorique à l'université de Caen sous Charles IX et Henri III.

\section{Théories et pratiques poétiques de l'humanisme (XVIe siècle) : la poésie (latine et française) des Parlementaires, dans la seconde moitié du XVI $I^{e}$ siècle en France}

L'année précédente, le directeur d'études avait orienté le travail du séminaire vers un nouveau type de poésie d'expression personnelle : les compositions des juristes, avocats ou conseillers aux Parlements en France dans la deuxième moitié du $\mathrm{XVI}^{\mathrm{e}}$ siècle. Suivant le grand exemple du chancelier de France Michel de L'Hospital (le directeur d'études prépare depuis quelques années l'édition de ses Poemata avec L. Petris, professeur à l'université de Neuchâtel), les robins envisagent l'écriture poétique non seulement - à la manière de Sénèque - comme un délassement formateur de l'esprit et indispensable au bon exercice de leur profession, mais même comme faisant partie de leurs devoirs (M. Fumaroli dans L'Âge de l'éloquence. Rhétorique et « res literaria » de la Renaissance au seuil de l'époque classique, Droz, 1980; rééd. Albin Michel, 1994, a montré que les Parlements se sentent responsables non seulement de la rédaction des lois, mais aussi de l'élaboration d'une rhétorique et d'une culture juridiques liées à la morale); ils sont également conscients que leurs recueils de poèmes de circonstance peuvent se révéler un moyen utile pour construire leur persona publique, voire pour gravir les échelons de leur carrière. L'Hospital réserve ainsi, lorsqu'il décrit les diverses sortes de poètes, un statut particulier aux parlementaires poètes, intermédiaire entre les poètes de profession, liés à l'humanisme, comme Jean Salmon Macrin ou Mellin de Saint-Gelais, qui sont bien souvent aussi des poètes de cour, et les simples amateurs de vers. Cette année la conférence a porté sur les Poemata d'Étienne de La Boétie, publiés dans une édition posthume par Montaigne, ainsi que ses poèmes français, en 1571, accompagnés d'une préface ambiguë de l'auteur des Essais, qui ne rend guère hommage au talent de son grand ami. Dans un premier temps, on a présenté le recueil en le comparant avec un autre recueil de poésies latines publié la même année, justement par le cousin de La Boétie, Guillaume de Calvimont. Les Silvae de Calvimont avaient fait l'objet du séminaire de l'année précédente. Calvimont, enfant bâtard d'une famille de robins en pleine ascension, avocat au Parlement de Bordeaux, 
puis au Parlement de Paris, a conçu ce volume pour mettre en valeur ses relations (il fréquente le salon des Deloynes-Morel, en même temps de L'Hospital, Macrin et la jeune Pléiade; il a des accointances avec la cour comme avec de hauts magistrats), pour exalter sa famille tout en réglant ses comptes avec son jeune frère légitime et héritier prioritaire de leur père et pour se constituer, comme bien des parlementaires, la persona d'un sage frotté de stoïcisme. Dans son recueil les registres poétiques se mêlent de façon très variées, faisant alterner les énigmes de cour, les jeux rhétoriques virtuoses, les badinages amicaux ou familiaux avec des méditations plus graves. É. de La Boétie, bien né, est entré, lui, par la grande porte dans le milieu de la magistrature, contrairement à Montaigne, dont l'ascension a été plus modeste; il se pose d'emblée dans ses Poemata à la fois comme un poète accompli qui connaît bien Macrin et pratique comme lui le culte de l'amour conjugal, et comme un proficiens, dessinant, grâce à la structure du recueil, un itinéraire spirituel d'essence stoïcienne également. Il offre de ses relations avec Montaigne une image (toujours colorée de stoïcisme) assez différente de celle qui ressort des Essais (rappelons que le directeur d'étude avait posé les fondements de cette recherche il y a quelques années lors d'un colloque sur La Boétie tenu à Duke University ${ }^{1}$, qui avait jeté en général une lumière nouvelle, moins idyllique, sur les rapports des deux hommes) : Montaigne, pourtant un peu plus âgé que lui, y apparaît comme un ami facilement en proie aux passions de la jeunesse que le sage La Boétie tente, tant bien que mal, de remettre sur le juste chemin. Par ailleurs, La Boétie fait preuve d'une maîtrise assez remarquable de la métrique et de l'intertexte poétique antique, d'une inventivité et d'une sensibilité qui dément les critiques que son ami formule dans le paratexte de l'édition posthume : il est fort probable que La Boétie avait bel et bien prévu et souhaité une édition de ses poèmes, qu'il avait sans doute, pour une grande part, déjà rassemblés et mis en ordre lui-même. En cours de séminaire on a pu étudier en détail, outre la structure du recueil, les textes liminaires en prose, la série des quatre premières pièces métapoétiques, et les trois pièces consacrées à Montaigne (I, III et XX).

\section{II. Édition, traduction et commentaire de textes humanistes.}

John Nassichuk (professeur à l'université de Western Ontario, Canada) : Les Poemata (Caen, 1600) de l'humaniste Jean Rouxel de Breteuil

L'humaniste Jean Rouxel (1530-1586) fut professeur de droit et de rhétorique à l'université de Caen sous Charles IX et Henri III. Excellent latiniste, il fut également un poète admiré, comme l'indique son biographe, Jacques de la Cahaignes, professeur royal de médecine à Caen, qui prononça son oraison funèbre. Rouxel est l'auteur de nombreuses œuvres. Après plusieurs séances traitant de la vie et de la carrière de Rouxel, cette partie du séminaire a été consacrée, sous la direction de J. Nassichuk, aux préliminaires de l'édition de certaines pièces poétiques. On a d'abord examiné rapidement le dialogue politique intitulé Deploratio Status Galliae (seconde moitié

1. P. Galand-Hallyn, « Les “essais” latins d’Étienne de La Boétie (Poemata, 1571) », dans M. Tetel (éd.), Étienne de La Boétie, Sage révolutionnaire et poète périgourdin, actes du colloque international de Duke University, 26-28 mars 1999, Paris, Champion, 2004, p. 123-156. 
du règne de Charles IX); ce poème dialogué selon une tradition littéraire qui remonte à Alain Chartier (Quadrilogue invectif, 1422), composé de 243 hexamètres dactyliques, met en scène des figurations des divers états sociaux et une allégorie de la Paix : Ecclesiastes (L'Homme d'Église), Patritius (Le Noble), Plebeius (Le Paysan), Astraea (Déesse de la Paix), qui réfléchissent ensemble aux motivations politiques des guerres de religion. On s'est ensuite intéressé à la paraphrase latine (cinq plaintes en distiques élégiaques) des Lamentations, l'antique plainte sur la destruction de Jérusalem devenue topos de la plainte civile, que Rouxel a publiée en 1568 (l'édition originale est aujourd'hui introuvable). J. Nassichuk a situé ce travail dans la vogue des Lamentations au $\mathrm{XVI}^{\mathrm{e}}$ siècle, a cherché à en dégager les inflexions propres à Rouxel avant de comparer cette réécriture avec le dialogue mentionné précédemment. Puis on en est venu à Rouxel poète des concours, des puys ou palinods (podia); ces concours de poésie étaient organisés, en l'honneur de la Vierge Marie, dans plusieurs villes de France; Rouxel a surtout fréquenté les puys de Rouen et, chose assez rare, a composé en latin (dans les années 1570); il a remporté de très nombreux prix, dont les circonstances sont précisées dans les marginalia des éditions (posthumes) de 1600 et 1636 (édition élargie) à Rouen. Plusieurs pièces ont été éditées, traduites et commentées lors du séminaire, qui témoignaient de la large culture littéraire de l'auteur, en vernaculaire comme en latin, de sa bonne connaissance de l'encyclopédisme antique comme de la poésie de la Latinité d'argent en particulier et d'un sens réel de l'inuentio.

Deux séances du séminaire ont été pour finir consacrées à des conférences : le professeur Dirk Sacré de l'Université catholique de Louvain (Belgique), spécialiste de néo-latin humaniste mais aussi plus tardif, est venu présenter un riche panorama de l'activité poétique en latin du XVII ${ }^{\mathrm{e}}$ au XIX ${ }^{\mathrm{e}}$ siècle, en illustrant son propos de l'analyse de plusieurs textes. Tristan Vigliano, maître de conférences à l'université Lyon-2, est venu présenter son livre : Humanisme et juste milieu au siècle de Rabelais. Essai de critique illusoire, Paris, Les Belles Lettres, 2009. 